\title{
Idiopathic mesenteric phlebosclerosis: clinical and CT imaging characteristics
}

\author{
Jian Ding, Weiqiang Zhang, Lizhang Wang, Zefeng Zhu, Jia Wang, Jianbing Ma \\ Department of Radiology, the First Hospital of Jiaxing, The Affiliated Hospital of Jiaxing University, Jiaxing, China
}

Correspondence to: Jianbing Ma, MD. Department of Radiology, the First Hospital of Jiaxing, The Affiliated Hospital of Jiaxing University, 1882, the South Zhonghuan Road, Jiaxing, China. Email: 413947372@qq.com.

Background: The aim of our study was to evaluate the clinical characteristics and computed tomography (CT) imaging findings of idiopathic mesenteric phlebosclerosis (IMP).

Methods: From January 2013 to May 2019, the clinical data of 10 patients diagnosed with IMP were analyzed retrospectively. Computed tomography angiography (CTA) and colonoscopy were performed in all 10 patients. All CT imaging findings were evaluated by three radiologists, including the form and distribution of calcification, the bowel's thickness, and the surrounding fat gap. The calcification score was calculated according to the extent of the involved mesenteric veins. The colonic wall thickness was defined as the average value of the thickest and thinnest regions of the intestinal wall. The correlation between the calcification scores and the colonic wall thickness was analyzed using Spearman's correlation analysis.

Results: All 10 patients were male with an average age of 59.6 years (range, 51-83 years). The average smoking index was 712 (range, 0-1,800). Among them, 7 patients had a history of long-term excessive daily intake of medicinal liquor or Chinese herbal medicine. Clinical symptoms of abdominal pain, diarrhea, bloating, and nausea were found. Colonoscopy showed dark purple discolorations of the edematous mucosa, engorged blood vessels, extensive erosion, ulceration, and multi-focal nodular surface in all patients. CT demonstrated colonic wall thickening, calcification along the mesenteric vein, and blurry surrounding fat gap in all 10 patients. Mesenteric venous calcification involved the terminal ileum, the ascending and transverse colon in all patients, and the descending colon and sigmoid colon's involvement in two patients. A total of 33 segments of the intestinal wall were involved. The median calcification score was 6 points, the mean thickness of the colonic wall was $10.73 \pm 3.22 \mathrm{~mm}$, and there was no significant correlation $(\mathrm{P}=0.782)$ between calcification score and thickness of the colonic wall.

Conclusions: The main features of IMP are mesenteric venous calcification, colonic wall thickness, and pericolic fat stranding, and there is no correlation between calcification score and colonic wall thickness. Therefore, CT imaging combined with colonoscopy can improve the diagnostic accuracy of IMP.

Keywords: Idiopathic mesenteric phlebosclerosis (IMP); calcification; CT

Submitted Feb 18, 2020. Accepted for publication Oct 09, 2020.

doi: 10.21037/qims-20-301

View this article at: http://dx.doi.org/10.21037/qims-20-301

\section{Introduction}

Idiopathic mesenteric phlebosclerosis (IMP) is a rare disease characterized by venous calcification extending from the colonic wall to the mesentery. IMP's etiology may be a chronic enteritis change caused by venous return obstruction without thrombosis and inflammation. The histological findings of IMP are as follows (1): (I) fibrosis thickening with calcification and luminal stenosis in the vein wall; (II) collagen deposition around the vessel in the mucosa; (III) foamy macrophages in the subserosa of the small vessel wall; (IV) no thrombosis in the blood vessel; (V) no active inflammation in the vascular lamina propria. The 
main clinical manifestations of IMP are often non-specific, including abdominal pain, nausea, and diarrhea. Therefore, it is easily misdiagnosed at first presentation. Mesenteric phlebosclerosis was first reported in Japan by Koyama et al. in 1991 (2), and formally named IMP by Iwashita et al. in 2003 (1). Subsequently, it was mainly reported in case reports, most of which were distributed across Southeast Asian countries such as Japan, China, and South Korea. There has been little research that has focused on IMP's imaging manifestations on computed tomography (CT) with a relatively large cohort of patients. Therefore, our study aimed to evaluate IMP's clinical and imaging features in CT imaging, with the largest series of cases reported in the literature.

\section{Methods}

\section{Patients and clinical features}

Ten patients were diagnosed with IMP from January 2013 to May 2019. All patients underwent computed tomography angiography (CTA) and colonoscopy. The patients' lifestyle factors and comorbidities were recorded. Among them, 9 patients were treated with conservative treatment, and 1 patient underwent surgical operation for intestinal obstruction.

The inclusion criteria were as follows: (I) patients with IMP confirmed by clinical diagnosis or pathology; (II) all patients underwent CTA and colonoscopy; (III) all patients had complete clinical data, including age, sex, medical history, and laboratory tests; (IV) CTA images were complete and processed after volume reconstruction (VR) and multiplanar reconstruction (MPR) recombination.

\section{Imaging acquisition and analysis}

CT images were acquired using SOMATOM Emotion 16, SOMATOM Definition AS+ and TSHIBA Aquilion ONE. All patients were asked to undergo a solid food fast for 12 hours before the examination, and drink 2,000 mL water mixed with mannitol 60 minutes before the scan. All patients underwent CTA of the mesenteric vessel after the administration of $100 \mathrm{~mL}$ of iodinated contrast medium. The scan range was from the phrenic apex to pubic symphysis level with a slice thickness and $5 \mathrm{~mm}$ interval. The phases of enhancement included arterial (1720 seconds) and venous phase (28-31 seconds). Nonionic contrast material (ioversol, $100 \mathrm{~mL}, 300 \mathrm{mg} / \mathrm{mL}$ ) was administered intravascularly using an automated injector at a rate of $4 \mathrm{~mL} / \mathrm{s}$ during a single breath-hold phase.

CT images were independently analyzed on the picture archiving and communication system (PACS) workstation by three radiologists experienced in gastrointestinal radiology (5, 10, and 12 years of experience, respectively). The degree of intestinal wall enhancement (mild or significant) was recorded on CT (arterial and venous phase) images and compared with the normal veins and muscles (it was considered mild or significant enhancement when the CT value of the intestinal wall was below muscle or higher than the vein) (3). The following characteristics on CT images of IMP were evaluated: (I) pericolic fat stranding; (II) cirrhosis; (III) splenomegaly; (IV) portal hypertension; (V) retroperitoneal lymph node.

MPR and VR were used to evaluate the images. The intestinal wall thickness was measured at the 3 thickest parts of each intestinal segment, and the average value was taken. Abnormal wall thickening was defined as when the dilatation of the colonic segment was greater than $20 \mathrm{~mm}$ and the thickness of the intestinal wall was greater than $5 \mathrm{~mm}$, or when the dilatation of colonic segment was more than $40 \mathrm{~mm}$ and the thickness of the intestinal wall was greater than $3 \mathrm{~mm}$ (4).

According to the mesenteric vein's anatomy, it was divided into 5 tributaries: ileocecal vein, right colon vein, middle colon vein, left colon vein, and sigmoid colon vein. Again, each tributary was divided into four segments: the straight vein, the marginal vein, the proximal half of the main branch of the mesenteric vein, and the distal end of the main branch. When calcification involved the above 4 venous segments, calcification scores were defined as 1,2, 3, and 4 , respectively (5). The total calcification score of each tributary of the mesenteric vein was the sum of 4 segments. Also, the colonic wall thickness corresponding to the 5 tributary veins and other imaging features related to IMP were recorded.

\section{Statistical analysis}

The inter-reader agreement for the calcification score and the colonic wall thickness were analyzed using the intraclass correlation coefficient (ICC). The consistency range of Landis and Koch was used as the reference standard for verification (6). The agreement was defined excellent (ICC $\geq 0.75$ ), moderate $(0.4<$ ICC $<0.75)$, and poor (ICC $\leq 0.4$ ). Data following the normal distribution were expressed as mean \pm standard deviation, and other data was represented 
Table 1 Clinical characteristics of patients with idiopathic mesenteric phlebosclerosis (IMP)

\begin{tabular}{|c|c|}
\hline Parameters & $\begin{array}{l}\text { Number of patients } \\
\qquad(\mathrm{n}=10)\end{array}$ \\
\hline \multicolumn{2}{|l|}{ Sex } \\
\hline Male & 10 \\
\hline Female & 0 \\
\hline Age at enrollment (years) & 59.6 (range, 51-83) \\
\hline Smoking index & $\begin{array}{l}9 \text { (average } 712 \text {, } \\
\text { range } 1-1,800 \text { ) }\end{array}$ \\
\hline Taking Chinese herbal medicine & 7 \\
\hline Hypertension & 5 \\
\hline Schistosoma & 3 \\
\hline Alcoholic liver disease & 1 \\
\hline HBV & 1 \\
\hline Rheumatism & 1 \\
\hline Other (kidney transplant) & 1 \\
\hline \multicolumn{2}{|l|}{ Symptom } \\
\hline Abdominal pain & 7 \\
\hline Diarrhea & 3 \\
\hline Abdominal distension & 2 \\
\hline Nausea and vomiting & 2 \\
\hline \multicolumn{2}{|l|}{ Laboratory examination } \\
\hline CRP (normal value 0-8 mg/L) & $7(+)$ \\
\hline D-D (normal value $0-500 \mathrm{ng} / \mathrm{mL}$ ) & $6(+)$ \\
\hline OB & $6(+)$ \\
\hline AFP (normal value $0-7 \mathrm{ng} / \mathrm{mL}$ ) & $2(+)$ \\
\hline CEA (normal value $0-5 \mathrm{ng} / \mathrm{mL}$ ) & $2(+)$ \\
\hline
\end{tabular}

+, abnormal. CPR, C-reactive protein; D-D, D-dimer; OB, occult blood; AFP, alpha-fetoprotein; CEA, carcinoembryonic antigen.

by median. The relationship between the calcification scores and the colonic wall thickness was calculated using Spearman's rank correlation coefficient. Statistical analysis was performed using SPSS software (version 18.0). A P value of $<0.05$ was considered statistically significant.

\section{Ethics approval}

The Ethics Committee approved this retrospective study of the First Hospital of Jiaxing. The informed consent was waived.

\section{Results}

\section{Patient clinical data}

The clinical characteristics of the 10 patients are presented in Table 1. Among the 10 patients, 7 patients presented with recurrent and worsening right upper quadrant pain, leading to hospitalization in our hospital. Other symptoms included diarrhea, abdominal distention, and nausea. Regarding medical history, the number of patients who had a history of smoking and used medicinal liquor or Chinese herbal medicines were 9 and 7, respectively. Moreover, various chronic diseases such as hypertension (5/10), schistosomiasis (3/10), rheumatism (1/10), hepatitis B (1/10), and alcoholic liver disease $(1 / 10)$ were reported as underlying disorders, including 1 patient who took immunosuppressants for 5 years due to kidney transplantation. Laboratory examinations showed that C-reactive protein, D-dimer, and tumor index were positive in 7,6 and 2 patients, respectively. Six patients were positive for fecal occult blood. Three patients with schistosomiasis had all been cured, and there were no symptoms of portal hypertension without the abnormality of white blood cell count, coagulation function, and hemoglobin level.

\section{Endoscopic view and histopathology}

Dark purple colored, rough, multiple erosions and ulcers of the mucosa were found in all patients (Figure 1). Nodular changes on the colon's surface, a stiff colonic wall, and disappearance of the colonic pouch were found in 3 patients. Gross specimen characteristics of the patient who underwent surgery included the mucosa of the distal ileum and ileocecal area appearing grey-purple, multiple convoluted blue-purple vessels, sclerosis nodules were found on the serosal surface, and thickening and calcification of the colonic wall (Figure 2A). The histopathological features included the extensive thickening of the colonic wall of the submucosal layer with collagen fibers. Thickening of the vascular wall in the submucosa was found. Calcification, collagen fibers, and osteoblasts in the venous wall also were found in the patient (Figure 2B,C,D).

\section{CT imaging findings}

The CT imaging characteristics of the 10 patients are presented in Table 2. A total of 33 segments of the colonic wall were involved. The lesions were mainly located in the terminal ileum, ascending and transverse colon (each of the 

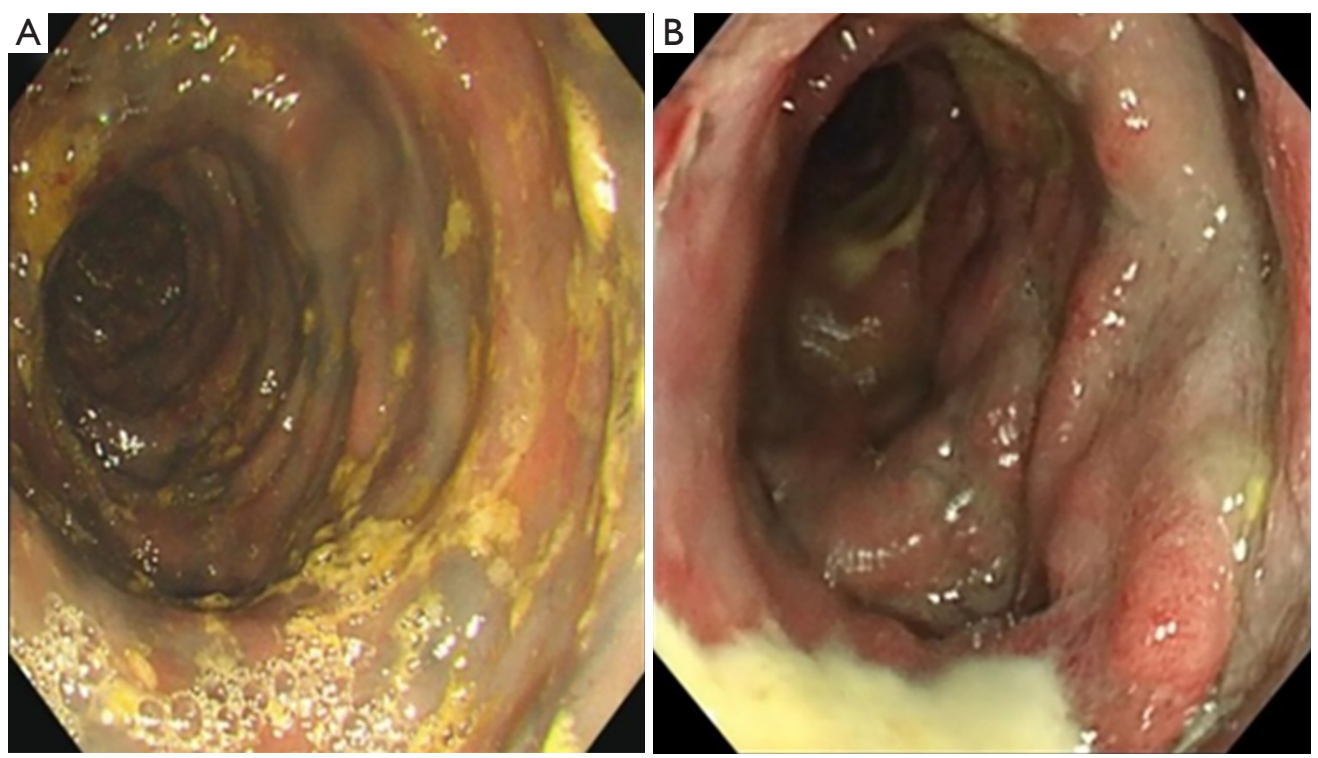

Figure 1 Idiopathic mesenteric phlebosclerosis (IMP) in a 57-year-old man. Nodular changes and a dark purple mucosal surface were found in the ascending colon (A). Large ulcers were found in the transverse colon (B).
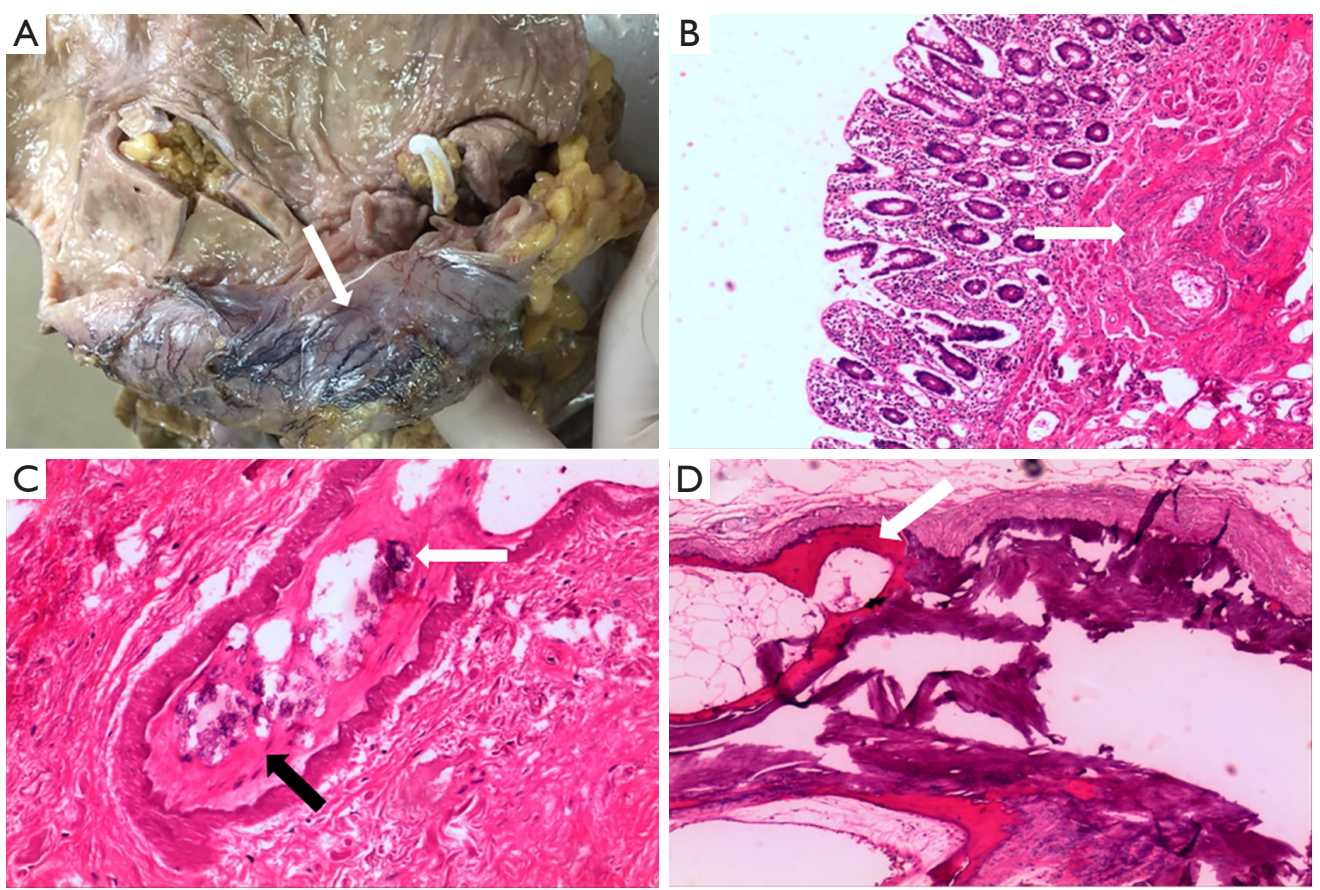

Figure 2 Idiopathic mesenteric phlebosclerosis (IMP) in a 59-year-old man who underwent surgery. (A) Multiple tortuous blue-purple vessels and sclerosis nodules in the ileocecal junction (white arrow). Purplish gray in the mucosal surface of the ileocecal region; (B) marked thickening of the vascular wall (white arrow) in the submucosa $(\mathrm{HE} \times 40)$; (C) calcification (white arrow) and collagen fibers (black arrow) in the venous wall $(\mathrm{HE} \times 100)$; (D) osteoblasts (white arrow) in the venous wall $(\mathrm{HE} \times 200)$. 
Table 2 The CT imaging characteristics of idiopathic mesenteric phlebosclerosis (IMP) patients

\begin{tabular}{|c|c|c|c|c|c|c|c|c|}
\hline Patient & Sex & $\begin{array}{c}\text { Age } \\
\text { (years) }\end{array}$ & Distribution of calcification & $\begin{array}{l}\text { Colonic wall thickness }(\mathrm{mm}) / \\
\text { calcification score }\end{array}$ & $\begin{array}{l}\text { Pericolic fat } \\
\text { stranding }\end{array}$ & $\begin{array}{l}\text { Intestinal } \\
\text { obstruction }\end{array}$ & $\begin{array}{l}\text { SMA and } \\
\text { IMA }\end{array}$ & $\begin{array}{c}\text { Degree of intestinal } \\
\text { wall enhancement }\end{array}$ \\
\hline 1 & M & 60 & $\begin{array}{l}\text { Ileocecum, right, } \\
\text { middle, left }\end{array}$ & $12.56 / 9.11$ & $(+)$ & $(-)$ & $(-)$ & Mild \\
\hline 2 & M & 51 & Ileocecum, right, middle & $10.60 / 6.92$ & $(+)$ & $(+)$ & $(-)$ & Mild \\
\hline 3 & M & 56 & Ileocecum, right, middle & $13.23 / 6.89$ & $(+)$ & $(-)$ & $(-)$ & Mild \\
\hline 5 & M & 57 & $\begin{array}{l}\text { Ileocecum, right, } \\
\text { middle, left, sigmoid }\end{array}$ & $8.26 / 7.86$ & $(+)$ & $(-)$ & $\begin{array}{l}(-) \\
(-)\end{array}$ & $\begin{array}{l}\text { Mild } \\
\text { Mild }\end{array}$ \\
\hline 6 & M & 61 & Ileocecum, right, middle & $7.90 / 3.33$ & $(+)$ & $(-)$ & $(-)$ & Mild \\
\hline 7 & M & 56 & Ileocecum, right, middle & $9.74 / 6.5$ & $(+)$ & $(-)$ & $(-)$ & Mild \\
\hline 10 & M & 58 & Ileocecum, right, middle & $9.83 / 4.78$ & $(+)$ & $(-)$ & $(-)$ & Mild \\
\hline
\end{tabular}

+ , positive; -, negative. IMP, idiopathic mesenteric phlebosclerosis; SMA, superior mesenteric artery; IMA, inferior mesenteric artery.

region was $30.3 \%)$, the descending colon $(6.1 \%)$, and the sigmoid colon $(3.0 \%)$.

Our study showed varying degrees of colonic wall thickening and calcification along the mesenteric vein and stiffness of the intestinal wall and exudation. After injection of a contrast agent, the main trunk and branches of the superior and inferior mesenteric artery were displayed, and there was no filling defect in the lumen. Intestinal mucous membrane enhancement was observed in the arterial and venous phase. Meanwhile, mesenteric torsion, intestinal tumors, and abdominal hernias were not found. In the 3 patients with schistosomiasis, hyperdense lineal shadows were shown in the liver on CT images, but cirrhosis and splenomegaly were not associated with this. No enlarged lymph nodes were found in the hilar of the liver or the retroperitoneum. A small amount of pelvic fluid was seen in the patient who underwent kidney transplantation. Pericolic fat stranding and intestinal obstruction were found in 9 patients.

One patient underwent plain abdominal radiograph and mesenteric artery CTA twice because of the symptom progression during follow-up. Plain abdominal radiograph and $C T$ scans all showed that the range of mesenteric vein calcification increased (Figure $3 A, B, C, D, E$ ), and there was no significant thickening of the intestinal wall compared to the previous CT images.
There was excellent inter-reader agreement [ICC $=0.883,0.886,95 \%$ confidence interval $(0.792-0.939)$, $(0.797-0.940)$, respectively] for assessing the calcification score and thickness of the intestinal wall. The calcification score's median value was 3 points, and the mean thickness of the intestinal wall was $10.90 \pm 3.70 \mathrm{~mm}$. There was no significant correlation $(\mathrm{P}=0.782)$ between calcification score and the colonic wall (Figure 4).

\section{Treatments and outcomes}

All 9 patients were hospitalized and received conservative treatment such as anti-inflammatory agents, improvement of microcirculation, acid suppression, and pain relief, and the symptoms were effectively relieved. Meanwhile, they were asked to stop drinking medicinal liquor, and received a low-residue diet. One patient underwent surgical treatment due to ineffective conservative treatment, and the condition presented as progressive aggravation. During the operation, the terminal ileum wall was significantly thickened, and the surface was purplish black accompanied by poor elasticity and a necrotic state. Band signs and mesenteric torsion were observed, and multiple tortuous blue-purple vessels were found in the submucosa. Unfortunately, he eventually died of sepsis. 

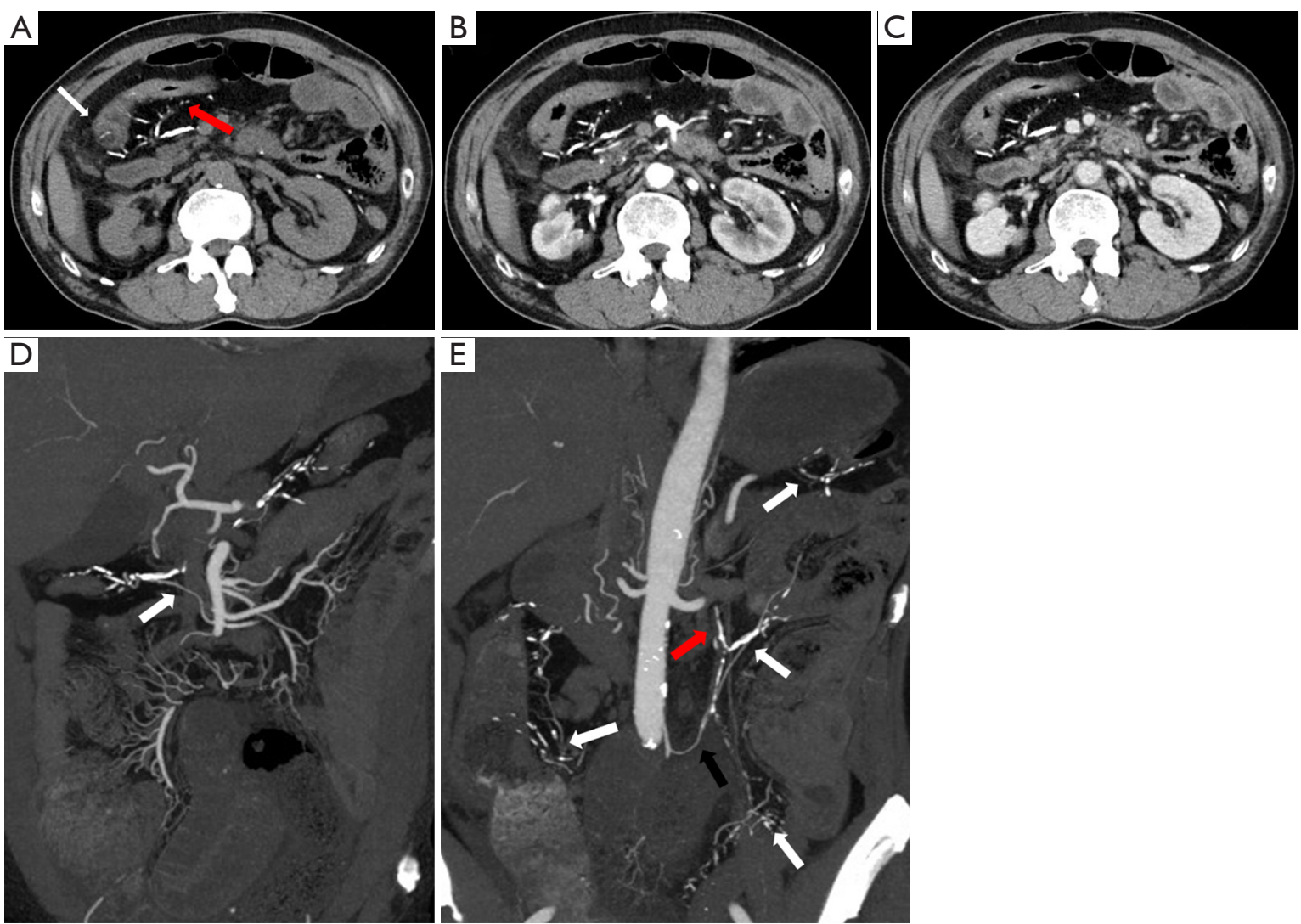

Figure 3 Idiopathic mesenteric phlebosclerosis (IMP) in a 59-year-old man who underwent surgery. He was admitted to our hospital for right lower quadrant pain. (A) Unenhanced CT image showed middle colonic wall thickening (white arrow) with calcification (red arrow) of the superior mesenteric vein involving the distal end of the main branch. Blurry surrounding fat gap hepatorenal crypt effusion was seen in the surrounding region; (B) arterial phase showed the uniform enhancement of the intestinal wall; (C) venous phase showed the continuous uniform enhancement of the colonic wall; (D) the transverse colonic branch of the superior mesenteric artery appeared smooth (arrow) without stenosis and calcification on the image of maximum intensity projection (MIP); (E) calcification was seen throughout the colon vein (white arrow), and the distal end of the main branch of the inferior mesenteric vein (red arrow) was involved. The trunk and branches of the inferior mesenteric artery were smooth without stenosis.

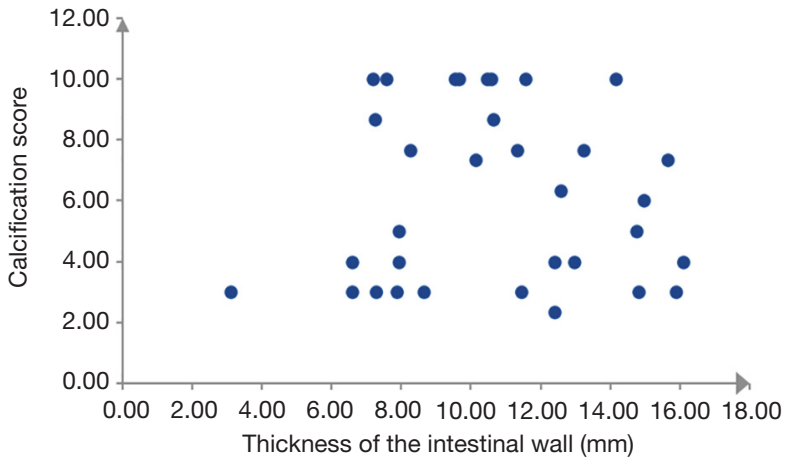

Figure 4 The dispersion diagram depicting the correlation between calcification score and the thickness of the intestine wall $(\mathrm{P}=0.782)$.

\section{Discussion}

Our results showed that the IMP characteristics of intestinal wall and mesenteric vein calcification can be clearly shown on CTA images, and the correlation between calcification degree and colorectal wall thickening can also be effectively evaluated. However, there was no significant relationship between calcification scores and colonic wall thickening.

IMP is also known as phlebosclerotic colitis or mesenteric venous sclerosis. It is a rare disease characterized by the diffuse thickening of the colonic wall with calcification in the venous wall along the straight and marginal vein. To the best of our knowledge, the pathogenesis of IMP is still unclear. According to previous reports $(7,8)$, immunological 
abnormality, hypercoagulopathy, and hypertension may contribute to IMP's etiology. Recently, it has been suggested that herbal medicine may play an important role in the pathogenesis of IMP, such as sanshishi and its metabolite $(9,10)$, and $70 \%$ of patients also had a history of taking herbal medicines in our study. Otherwise, hypertension, diabetes, schistosomiasis, cirrhosis, and rheumatism are thought to be related to this disease, but only a few cases have been reported so far, requiring a larger data set to support this finding. All the patients in our study had a history of residents over 40 years with a mean age of 59.6 years, which is in line with the literature $(11,12)$.

In addition to taking herbal medicine, the occurrence and location of lesions are related to the colon's physiological mechanisms. Although it is similar to other ischemic bowel diseases, long-term chronic hypoxia of the intestinal mucosal venous muscle layer can damage the vein, and IMP can cause calcification and sclerosis formation of the venous wall. The damaged veins develop reactive hyperplasia, leading to vascular thickening, occlusion, and ischemia. Finally, it causes the thickening of the intestinal wall. Also, the absorption of water and partial digestion mainly occurs in the ileocecum and ascending colon, and the roundtrip movement of haustrum causes repeated absorption of sitotoxin in the ileocecum and ascending colon. Therefore, the right colon is frequently involved. On the other hand, the ascending colon's movement direction is opposite to the gravity of food, which prolongs the retention time of food in the right colon. In our study, the lesions involved the ileocecum, and the ascending and transverse colon in all patients.

The early symptoms of IMP may be atypical or absent. As the disease progresses, abdominal pain, abnormal stool, or intestinal obstructions may manifest. The most common symptoms in our study were abdominal pain $(70 \%)$ and diarrhea $(30 \%)$. In addition, laboratory tests were also nonspecific. Highly sensitive C-reactive protein (70\%), positive plasma D-dimer (60\%), and fecal occult blood testing $(60 \%)$ were found in our study. Therefore, it is difficult to differentiate among these inflammatory and ischemic bowel diseases due to the similar clinical symptoms and pathology markers.

Concerning the previous literature $(13,14)$, and in combination with our study, the CT imaging features of IMP can be summarized as follows: (I) lesions mainly occur in the colon, especially in the ileocecum, ascending colon, and transverse colon; (II) the calcification of the superior mesenteric vein is a characteristic manifestation on CT images, and is found in the right colon more frequently, shown as strips and aligned with the vessel and perpendicular to the intestine. MPR and CTA are favorable for displaying the morphology of calcification; (III) the colonic wall is mainly thickened with slow peristalsis and pericolic fat stranding, and haustrum cannot be found in some cases; (IV) there is no thrombosis in the main trunk and branches of the superior and inferior mesenteric artery, and mesenteric torsion, intestinal tumors, and abdominal hernias are not found.

The correlation between venous calcification score and intestinal wall thickness was not significant $(\mathrm{P}>0.05)$ in our study suggesting that the thickness of the intestinal wall would not thicken with the increasing calcification score of the mesenteric vein. Therefore, we speculate that the pathological mechanisms are venous reflux disorder and decreased oxygenation due to the mesenteric vein wall's calcification, leading to a decrease in bowel dynamics, food residue, and air retention in the intestine, resulting in a thinner intestinal wall. Also, we speculate that the extent of mesenteric vein calcification is related to the severity of the disease, and a larger data set is needed to verify this correlation.

In our study, 2/10 patients had increased calcification range during follow-up, and the clinical symptoms were also aggravated. However, the wall of the tube became thinner in varying degrees. One of the 2 patients underwent surgical treatment due to the increased intestinal obstruction. A microscopic examination of the removed colon tissue showed a thickened submucosa and venous wall, and different numbers of calcification and ossification of the venous media causing negative remodeling of vessels.

In our study, colonoscopy was performed in all 10 patients, which showed dark purple discolorations of the edematous mucosa, engorged blood vessels, and extensive ulcerations, similar to previous reports (15-17). IMP can be misdiagnosed as other ischemic bowel diseases due to the lack of awareness. In this study, 6 patients were misdiagnosed with ulcerative colitis. IMP should be mainly distinguished from the following diseases: (I) ulcerative colitis-the main symptoms are bloody diarrhea and abdominal pain, though multiple ulcers can be found in ulcerative colitis and IMP under colonoscopy. The colon walls in ulcerative colitis do not appear purple under colonoscopy and are rarely associated with wall thickening and mesenteric vein calcification (18); (II) schistosome intestinal disease-usually in patients who have resided in a schistosome distribution area. Involves the descending 
and sigmoid colon, characterized by calcified nodules which accumulate only in the mucous layer without involving the main mesenteric vein (19); (III) ischemic bowel diseasemainly caused by acute mesenteric artery ischemic. The position of the lesion is unfixed without the calcification of the mesenteric vein. Presence of a thickened and swollen intestinal wall with the thrombus in the lumen of the mesenteric artery which can be seen in CT images $(20,21)$. Otherwise, intestinal obstruction is also observed in some severe cases.

The present study has several limitations. First, it was a retrospective analysis, and a small number of IMP patients were included owing to its extremely low incidence. Second, although three radiologists measured the intestinal wall's data on the PACS workstation, it needed to be further improved due to uncontrollable factors. Additionally, there was no long-term follow-up to evaluate the correlation between calcification score and surgery in our study. Finally, since most of the patients received conservative treatment in this study, only 1 patient underwent surgical treatment, and there was not enough histopathological evidence for the diagnosis of IMP.

\section{Conclusions}

IMP is a rare disease owing to the atypical clinical manifestations and laboratory test markers. As shown in our study, the morphological features of mesenteric vein calcification can be clearly observed, and the correlation between calcification degree and colonic wall thickening can also be effectively evaluated on CTA images. Dark purple colored colon and mesenteric calcification are the typical manifestations visualized on colonoscopy and CTA, respectively. Colonoscopy can also clearly show the changes of the mucosal layer of the colonic wall. Therefore, CTA combined with colonoscopy examination can effectively improve the diagnostic accuracy of IMP.

\section{Acknowledgments}

Funding: Jiaxing Key Disciplines (No.: 2019-fc-06).

\section{Footnote}

Conflicts of Interest: All authors have completed the ICMJE uniform disclosure form (available at http://dx.doi. org/10.21037/qims-20-301). The authors have no conflicts of interest to declare.
Ethical Statement: This study was approved by the Ethics Committee of the First Hospital of Jiaxing. The requirement for individual consent was waived by the committee because of the retrospective nature of the study.

Open Access Statement: This is an Open Access article distributed in accordance with the Creative Commons Attribution-NonCommercial-NoDerivs 4.0 International License (CC BY-NC-ND 4.0), which permits the noncommercial replication and distribution of the article with the strict proviso that no changes or edits are made and the original work is properly cited (including links to both the formal publication through the relevant DOI and the license). See: https://creativecommons.org/licenses/by-nc-nd/4.0/.

\section{References}

1. Iwashita A, Yao T, Schlemper RJ, Kuwano Y, Yao T, Iida M, Matsumoto T, Kikuchi M. Mesenteric phlebosclerosis: a new disease entity causing ischemic colitis. Dis Colon Rectum 2003;46:209-20.

2. Koyama N, Koyama H, Hanashima T. A case of rightsided constrictive ischemic colitis presenting with a chronic course. Stom. Intest (Tokyo) 1991;26:455-60.

3. Wittenberg J, Harisinghani MG, Jhaveri K, Varghese J. Mueller PR. Algorithmic approach to CT diagnosis of the abnormal bowel wall. Radiographics 2002;22:1093-107.

4. Wiesner W, Mortelé, Koenraad J, Ji H, Ros PR. Normal colonic wall thickness at $\mathrm{CT}$ and its relation to colonic distension. J Comput Assist Tomogr 2002;26:102-6.

5. Lin WC, Chen JH, Westphalen AC, Liao CH, Chen CH, Chen CM, Lin CH. The role of CT in predicting the need for surgery in patients diagnosed with mesenteric phlebosclerosis. Medicine (Baltimore) 2016;95:e5139.

6. Landis JR, Koch GG. The measurement of observer agreement for categorical data. Biometrics 1977;33:159-74.

7. Kitamura T, Kubo M, Nakanishi T, Fushimi H, Yoshikawa K, Taenaka N, Furukawa T, Tsujimura T, Kameyama M. Phlebosclerosis of the colon with positive anti-centromere antibody. Intern Med 1999;38:416-21.

8. Kusanagi M, Matsui O, Kawashima H, Gabata T, Ida M, Abo H, Isse K. Phlebosclerotic colitis: imaging-pathologic correlation. AJR Am J Roentgenol 2005;185:441-7.

9. Hoshino N, Hasegawa S, Hida K, Kawada K, Sakai Y. Right hemicolectomy for mesenteric phlebosclerosis potentially caused by long-term use of herbal medicine: A case report and literature review. Int J Surg Case Rep 
2016;24:191-4.

10. Shimizu S, Kobayashi T, Tomioka H, Ohtsu K, Matsui T, Hibi T. Involvement of herbal medicine as a cause of mesenteric phlebosclerosis: results from a large-scale nationwide survey. J Gastroenterol 2017;52:308-14.

11. Guo F, Zhou YF, Zhang F, Yuan F, Yuan YZ, Yao WY. Idiopathic mesenteric phlebosclerosis associated with longterm use of medical liquor: two case reports and literature review. World J Gastroenterol 2014;20:5561-6.

12. Chen W, Zhu H, Chen H, Shan G, Xu G, Chen L, Dong F. Phlebosclerotic colitis: Our clinical experience of 25 patients in China. Medicine (Baltimore) 2018;97:e12824.

13. Choi JM, Lee KN, Kim HS, Lee SK, Lee JG, Lee, SW, Lee OY, Choi HS. Idiopathic phlebosclerotic colitis: a rare entity of chronic ischemic colitis. Korean J Gastroenterol 2014;63:183-6.

14. Nomura K, Kikuchi D, Iizuka T, Yamada A, Furuhata T, Domon K, Yamashita S, Nakamura M, Matsui A, Mitani T, Ogawa O, Hoteya S, Kaise M. Idiopathic mesenteric phlebosclerosis associated with long-term use of Chinese herbs: a case report. Nihon Shokakibyo Gakkai Zasshi 2012;109:1567-74.

15. Hiramatsu K, Sakata H, Horita Y, Orita N, Kida A, Mizukami A, Miyazawa M, Hirai S, Shimatani A, Matsuda K, Matsuda M, Ogino H, Fujinaga H, Terada I, Shimizu

Cite this article as: Ding $\mathrm{J}$, Zhang W, Wang L, Zhu Z, Wang J, Ma J. Idiopathic mesenteric phlebosclerosis: clinical and CT imaging characteristics. Quant Imaging Med Surg 2021;11(2):763-771. doi: 10.21037/qims-20-301
K, Uchiyama A, Ishizawa S, Abo H, Demachi H, Noda Y. Mesenteric phlebosclerosis associated with long-term oral intake of geniposide, an ingredient of herbal medicine. Aliment Pharmacol Ther 2012;36:575-86.

16. Sasaki Y, Saito M, Koshiba Y, Zai H, Urita Y. Idiopathic mesenteric phlebosclerosis associated with herbal drugs presenting with asymptomatic fecal occult blood. J Gen Fam Med 2017;18:475-76.

17. Lee SM, Seo JW. Phlebosclerotic colitis: case report and literature review focused on the radiologic findings in relation to the intake period of toxic material. Jpn J Radiol 2015;33:663-7.

18. Prabhakar N, Kalra N, Bhasin DK, Rana SS, Gupta V, Singh R, Khandelwal N. Comparison of CT colonography with conventional colonoscopy in patients with ulcerative colitis. Acad Radiol 2015;22:296-302.

19. Lee RC, Chiang JH, Chou YH, Rubesin SE, Wu HP, Jeng WC, Hsu CC, Tiu CM, Chang T. Intestinal schistosomiasis japonica: CT-pathologic correlation. Radiology 1994;193:539-42.

20. Kim JS, Kim HJ, Hong SM, Park SH, Lee JS, Kim AY, Ha HK. Post-Ischemic Bowel Stricture: CT Features in Eight Cases. Korean J Radiol 2017;18:936-45.

21. Alpern MB, Glazer GM, Francis IR. Ischemic or infarcted bowel: CT findings. Radiology 1988;166:149-52. 